IBIMA Publishing

International Journal of Veterinary Medicine: Research \& Reports

http://www.ibimapublishing.com/journals/IJVMR/ijvmr.html

Vol. 2013 (2013), Article ID 204515, 2 pages

DOI: $10.5171 / 2013.204515$

Research Article

\title{
A Case of Tinea Pedis Caused by Trichophyton Tonsurans
}

\section{B.C. Carlsen and T. Menné}

Department of Dermato-Allergology, Copenhagen University Hospital Gentofte, Denmark

Correspondence should be addressed to: B.C. Carlsen; $\underline{\text { bccarlsen@dadlnet.dk }}$

Received 11 February 2013; Accepted 26 February 2013; Published 31 March 2013

Academic Editor: Roberto Arenas

Copyright (C) 2013 B.C. Carlsen and T. Menné. Distributed under Creative Commons CC-BY 3.0

\begin{abstract}
Tinea infections are very common skin disorders caused by dermatophytes that have the unique ability to invade tissue containing keratin. The specific aetiological agents vary geographically related to environmental and cultural factors. The commonest causes of tinea infections in Europe include Trichophyton rubrum, mentagrophytes et verrucosum and Epidermophyton floccosum. The typical dermatophytes responsible for tinea pedis are Trichophyton rubrum et mentagrophytes and Epidermophyton floccosum. We here present a case of tinea pedis with an unusual aetiological pathogen: Trichophyton tonsurans.
\end{abstract}

Keywords: Tinea pedis, Trichophyton tonsurans, dermatophytosis, fungal infection.

\section{Introduction}

\section{Case}

A 46 year old Danish Caucasian man was referred to a hospital dermatology department under the diagnosis of recurrent vesicular hand eczema. He had suffered from vesicular outbreaks located to the hands for three years prior to referral and had been treated with topical corticosteroids without any effect. He presented with bilateral severe hand eczema primarily dominated with vesicular changes located in the palmar region of the hands. No skin changes were present on the dorsal part of the hands or fingers. Similar vesicular changes were present on the soles and heels of the feet bilaterally along with hyperkeratosis, erythema and scaling.
The patient worked as a watchmaker and wore his own selfmade watches without developing any rash around the wrists. He was patch tested for type IV allergies including the metals chromate, nickel, palladium and cobalt. The patch test was negative. No sports activity or travel history was noted for this patient.

A conventional mycological examination of the feet was performed and myceliums were present in the microscopy. In the culture Trichophyton tonsurans was identified based on morphological features.

\section{Discussion}

This is a case of Trichophyton Tonsurans causing tinea pedis: an unusual aetiological pathogen for both the bodily and geographical region. Typically, T. Tonsurans causes tinea capitis ranging from tinea

Cite this Article as: B.C. Carlsen and T. Menné (2013), "A Case of Tinea Pedis Caused by Trichophyton Tonsurans," International Journal of Veterinary Medicine: Research \& Reports, Vol. 2013 (2013), Article ID 204515, DOI: 10.5171/2013.204515 
capitis carriage status to clinical infection (Havlickova B et al 2008). Besides tinea capitis, Trichophyton Tonsurans causes tinea corporis and a case of Majoccis granuloma and folliculitis decalvans due to Trichophyton tonsurans has also been described (Liao YH et al 1999, HryncewiczGwozdz A et al 2011). Trichophyton tonsurans has not been described as an aethiological factor for tinea pedis in Northen Europe before. Furthermore, T. Tonsurans in itself is an atypical aetiological fungal pathogen in Northern Europe. Even though, T. Tonsurans is the commonest cause of tinea capitis in the US, in most part of Europe it is a rare cause of tinea infections. Increases in T. tonsurans tinea capitis is, however, recently observed in both France and the United Kingdom primarily caused by immigration from Africa (Fuller LC et al 2009). The patient described in this case report also presented with an atypical combination of clinical symptoms related to both the moccasin and vesicular type of tinea pedis e.g. low grade inflammation on the feet but with accompanying dermatophytid reaction. The moccasin type of tinea pedis is characterized by low grade inflammation with diffuse hyperkeratosis, erythema, scaling and fissures on the plantar surfaces. Vesicular changes are not typical for this subtype. The vesicular type of tinea pedis presents with more severe inflammation with vesicles and bullae on the medial foot and is associated with the dermatophytid reaction. Antrophophilic fungi are the commonest cause of the moccasin type, whereas zoophilic fungi are the major cause of the vesicular type of tinea pedis. Trichophyton tonsurans is an antropophilic fungus. The atypical presentation of tinea pedis matches the atypical finding of Trichophyton tonsurans as an aetiological agent and may well explain why this patient was misdiagnosed for three years. It also highlights the importance of considering a fungal infection and dermatophytid reactions in patients who at first glance seem to suffer from hand eczema.

Atypical fungal pathogens may become more common in the Western part of the World with the increasing tourism and migration of people. Atypical fungal pathogens should be considered for atypical presentations of tinea pedis as well as be considered as a differential diagnosis when treating dermatoses that can mimick a tinea infection.

\section{References}

Fuller, L. C. (2009). "Changing Face of Tinea Capitis in Europe," Current Opinion in Infectious Diseases, 22(2) 115-8.

Havlickova, B, Czaika, V. A. \& Friedrich, M. (2008). "Epidemiological Trends in Skin Mycoses Worldwide," Mycoses, 51(Suppl 4) 2-15.

Hryncewicz-Gwozdz, A., BeckJendroscheck, V., Brasch, J., Kalinowska, K. \& Jagielski, T. (2011). "Tinea Capitis and Tinea Corporis with a Severe Inflammatory Response Due to Trichophyton Tonsurans," Acta Dermato-Venerologica, 91(6) 708-710.

Liao, Y. H., Chu, S. H., Hsiao, G. H., Chou, N. K., Wang, S. S. \& Chiu, H. C. (1999). "Majocci's Granuloma Caused by Trichophyton Tonsurans in a Cardiac Transplant Recipient," British Journal of Dermatology, 140(6) 1194-6. 